\title{
Criminologie
}

\section{La transition des jeunes femmes hors des centres de réadaptation} Mieux comprendre le rôle de l'agression sexuelle sur des mineures et des symptômes liés au trauma

\section{How do female adolescents transition out of residential care ?}

Towards a comprehensive understanding of child sexual abuse in relation to trauma-related symptoms

\section{La transición de las adolescentes afuera de los centros de rehabilitación \\ Hacia una mayor comprensión del abuso sexual del niño en relación con los síntomas del traumatismo}

\author{
Eveline Van Vugt, Nadine Lanctôt, Geneviève Paquette et Annie Lemieux
}

Volume 50, numéro 1, printemps 2017

L'agression sexuelle commise sur des mineurs : les victimes, les auteurs

URI : https://id.erudit.org/iderudit/1039799ar

DOI : https://doi.org/10.7202/1039799ar

Aller au sommaire du numéro

Éditeur(s)

Les Presses de l’Université de Montréal

ISSN

0316-0041 (imprimé)

1492-1367 (numérique)

Découvrir la revue

Citer cet article

Van Vugt, E., Lanctôt, N., Paquette, G. \& Lemieux, A. (2017). La transition des jeunes femmes hors des centres de réadaptation : mieux comprendre le rôle de l'agression sexuelle sur des mineures et des symptômes liés au trauma.

Criminologie, 50(1), 127-155. https://doi.org/10.7202/1039799ar

\section{Résumé de l'article}

Cet article souhaite apporter une contribution à l'étude des symptômes liés au trauma observés au début de l'âge adulte chez des jeunes femmes ayant été placées en centre de réadaptation au cours de l'adolescence et qui ont été victimes d'agression sexuelle avant l'âge de 18 ans. En vue de mieux comprendre l'expérience de l'agression sexuelle, les impacts d'un certain nombre de caractéristiques ont été étudiés : la sévérité et la diversité de l'expérience de l'agression sexuelle, la fréquence et la chronicité de l'agression sexuelle, la familiarité avec l'agresseur et l'âge à la première survenue de l'agression sexuelle. Méthode : L'échantillon est composé de 133 jeunes femmes adultes qui ont été placées en centre de réadaptation à l'adolescence. L'agression sexuelle a été évaluée rétrospectivement à l'aide d'une version adaptée du questionnaire sur l'agression sexuelle développé par Finkelhor, Hotaling, Lewis et Smith (1990). Les symptômes liés au trauma ont été évalués à l'aide de l'Inventaire des symptômes liés au trauma-2 (Brière, 2011). Résultats : Les jeunes femmes ayant vécu une agression sexuelle présentent des niveaux plus élevés de symptômes liés au trauma que celles qui n’ont pas été agressées sexuellement. De plus, les jeunes femmes ayant signalé une gravité (viol), une diversité et une persistance (chronicité) plus importantes d'agressions sexuelles, montrent des niveaux élevés de symptômes liés au trauma. Ces symptômes se manifestent par des pensées intrusives, des problèmes d'attachement et de l'évitement défensif. Les différences observées quant à la fréquence, à l'identité de l'agresseur et à l'âge de la survenue des agressions sexuelles sont moins discriminantes en ce qui concerne les symptômes liés au trauma. Conclusion : L'analyse des caractéristiques des agressions sexuelles confirme la nécessité d'aider les femmes ayant vécu des expériences persistantes et diverses d'agression sexuelle afin qu'elles puissent créer des relations fondées sur un sentiment de sécurité. 


\title{
La transition des jeunes femmes hors des centres de réadaptation
}

\author{
Mieux comprendre le rôle de l'agression \\ sexuelle sur des mineures et des symptômes \\ liés au trauma
}

\author{
Eveline Van Vugt ${ }^{1}$ \\ Professeure adjointe \\ Université d'Amsterdam \\ E.S.vanVugt@uva.nl \\ Nadine Lanctôt \\ Professeure agrégée \\ Université de Sherbrooke \\ Nadine.Lanctot@USherbrooke.ca \\ Geneviève Paquette \\ Professeure agrégée \\ Université de Sherbrooke \\ Genevieve.Paquette@USherbrooke.ca \\ Annie Lemieux \\ Statisticienne \\ Université de Sherbrooke \\ Annie.D.Lemieux@Sherbrooke.ca
}

\section{Article traduit de l'anglais par Fyscillia Ream}

RÉSUMÉ - Cet article souhaite apporter une contribution à l'étude des symptômes liés au trauma observés au début de l'âge adulte chez des jeunes femmes ayant été placées en centre de réadaptation au cours de l'adolescence et qui ont été victimes d'agression sexuelle avant l'âge de 18 ans. En vue de mieux comprendre l'expérience de l'agression sexuelle, les impacts d'un certain nombre de caractéristiques ont été étudiés: la sévérité et la diversité de l'expérience de l'agression sexuelle, la fréquence et la chronicité de l'agression sexuelle, la familiarité avec l'agresseur et l'âge à la première

1. Faculteit der Maatschappij- en Gedragswetenschappen, Universiteit van Amsterdam, Nieuwe Achtergracht 127, 1018 WS Amsterdam, Pays-Bas. 
survenue de l'agression sexuelle. Méthode: L'échantillon est composé de 133 jeunes femmes adultes qui ont été placées en centre de réadaptation à l'adolescence. L'agression sexuelle a été évaluée rétrospectivement à l'aide d'une version adaptée du questionnaire sur l'agression sexuelle développé par Finkelhor, Hotaling, Lewis et Smith (1990). Les symptômes liés au trauma ont été évalués à l'aide de l'Inventaire des symptômes liés au trauma-2 (Brière, 2011). Résultats: Les jeunes femmes ayant vécu une agression sexuelle présentent des niveaux plus élevés de symptômes liés au trauma que celles qui n'ont pas été agressées sexuellement. De plus, les jeunes femmes ayant signalé une gravité (viol), une diversité et une persistance (chronicité) plus importantes d'agressions sexuelles, montrent des niveaux élevés de symptômes liés au trauma. Ces symptômes se manifestent par des pensées intrusives, des problèmes d'attachement et de l'évitement défensif. Les différences observées quant à la fréquence, à l'identité de l'agresseur et à l'âge de la survenue des agressions sexuelles sont moins discriminantes en ce qui concerne les symptômes liés au trauma. Conclusion: L'analyse des caractéristiques des agressions sexuelles confirme la nécessité d'aider les femmes ayant vécu des expériences persistantes et diverses d'agression sexuelle afin qu'elles puissent créer des relations fondées sur un sentiment de sécurité.

MotS CLÉS - Agression sexuelle, symptômes liés au trauma, début de l'âge adulte, femmes, centre de réadaptation.

\section{Introduction}

À l'échelle mondiale, l'agression sexuelle commise sur des mineurs est considérée comme un grave problème de santé publique. Les taux de prévalence globaux indiquent qu'approximativement une fille sur cinq et un garçon sur treize ont été victimes de quelque forme d'agression sexuelle durant l'enfance, y compris le viol, l'atteinte à la pudeur ou l'exploitation sexuelle (Pereda, Guilera, Forns et Gomez-Benito, 2009; Stoltenborgh, Van IJzendoorn, Euser et Bakermans-Kranenburg, 2011). Depuis quelques années, on prend de plus en plus conscience et on s'inquiète davantage de la prévalence et des répercussions de l'agression sexuelle subie durant l'enfance chez les jeunes placés par les services de la protection de l'enfance. Comparativement aux jeunes recevant des traitements dans la communauté, les taux de prévalence d'agression sexuelle parmi les jeunes en centre de réadaptation sont deux fois plus élevés (Briggs et al., 2012), le taux étant plus élevé chez les filles, soit près de $60 \%$ (Collin-Vézina, Coleman, Milne, Sell et Daigneault, 2011; Connor, Doerfler, Toscano, Volungis et Steingard, 2004). Des taux similaires ont été observés dans les études portant sur des adolescentes mises sous garde en contexte judiciaire (Belknap et Holsinger, 2006; Goodkind, Ng et Sarri, 2004).

Il a été démontré que l'agression sexuelle peut être traumatisante et altérer les processus développementaux à court et à long terme (Hillberg, 
Hamilton-Giachritsis et Dixon, 2011; Maniglio, 2009). Des études ont montré que la nature interpersonnelle, intrusive et parfois chronique de l'agression sexuelle durant l'enfance, qui inclut l'abus de confiance et la manipulation (Finkelhor, 2008; Finkelhor, Omrod et Turner, 2009), altèrent les processus développementaux des enfants (D’Andrea, Ford, Stolbach, Spinazzola et Van der Kolk, 2012). Plus précisément, cette altération peut entraîner des symptômes spécifiques aussi connus sous le nom du trauma complexe (Cloitre et al., 2009; Herman, 1997). Les évènements liés au trauma complexe, survenant seuls ou en cooccurrence, comprennent entre autres l'agression sexuelle, émotionnelle ou physique, la négligence, le fait d'être témoin de violence conjugale et d'autres situations traumatisantes telles que la guerre, la captivité ou le trafic d'êtres humains (Wamser-Nanney et Vandenberg, 2013). Le trauma complexe réfère aussi bien à l'exposition prolongée de la victime aux évènements traumatiques dans un contexte interpersonnel qu'à l'impact de cette exposition et ses conséquences immédiates et sur le long terme (Cook et al., 2005; Wamser-Nanney et Vandenberg, 2013). Le trauma complexe peut se produire lorsque le traumatisme, à l'instar de l'agression sexuelle, 1) est répétitif ou chronique; 2) est commis par un proche ou un autre adulte significatif dans l'environnement de l'enfant; et 3) couvre une ou plusieurs phases vulnérables du développement (Courtois et Ford, 2009). Le trauma complexe s'étend au-delà des symptômes traditionnels du syndrome de stress post-traumatique et inclut des désordres de régulation de l'affect, de somatisation et de difficultés interpersonnelles (Brière et Scott, 2015; Cloitre et al., 2009; D'Andrea et al., 2012). Wamser-Nanney et Vandenberg (2013) ont montré que les enfants et les adolescents en recherche de traitement, exposés à un trauma complexe, présentent une constellation de symptômes différents de ceux qui sont exposés à des traumatismes non complexes (par ex.: traumatisme non interpersonnel aigu).

Dans cette perspective, les chercheurs et les praticiens reconnaissent la nécessité de concevoir et de mettre en œuvre des pratiques éclairées fondées sur le trauma (Covington et Bloom, 2006; Kerig et Schindler, 2013). Dans les milieux d'intervention où ces pratiques sont déjà à l'œuvre, le personnel est formé pour reconnaître un large éventail de manifestations du trauma complexe observé chez les jeunes et sait comment y répondre. Par exemple, on réalise des entrevues en profondeur avec les jeunes en vue de découvrir de possibles expériences de maltraitance et de négligence. Les expériences traumatiques sont éga- 
lement abordées dans le cadre de la planification du traitement où l'on considère les préférences du jeune relativement à l'aide dont il aura besoin à certains moments (par ex.: déterminer quel membre du personnel et de quelle manière le jeune doit être abordé lors d'une crise) (Lathma-Hummer, Dollard, Robst et Armstrong, 2010). En faisant participer le jeune à la résolution du problème, on encourage ainsi son autonomie.

Jusqu'à ce jour, les décisions de placement et de traitement dans un établissement d'accueil ont généralement été fondées sur des rapports officiels de maltraitance et de négligence, y compris l'agression sexuelle commise contre des enfants (Ministère de la Santé et des Services sociaux, 2007). Se fier uniquement aux rapports officiels pour déterminer la maltraitance subie par un enfant comporte le risque de sousestimer la situation, car seul un petit nombre de cas sera porté à l'attention des autorités officielles, et un nombre encore plus petit offrira des preuves suffisantes (Collin-Vézina, Daigneault et Hébert, 2013). Cela est particulièrement vrai dans les cas d'agression sexuelle, généralement cachés ou secrets. Ces sous-estimations systématiques, résultant des procédures de dépistage des services de protection de l'enfance, peuvent affecter le niveau et le type de soins que les jeunes reçoivent. Ainsi, s'appuyer sur des informations autorapportées additionnelles peut aider à bien déterminer les antécédents de maltraitance et de négligence du jeune (Hambrick, Tunno, Gabrielli, Jackson et Belz, 2014).

Les symptômes liés à l'agression sexuelle durant l'enfance peuvent non seulement se manifester directement après l'agression mais peuvent aussi persister à l'âge adulte (Fergusson, McLeod et Horwood, 2013; Van Vugt, Lanctôt, Paquette, Collin-Vézina et Lemieux, 2014). Les jeunes devant bientôt quitter leur milieu de placement, soit parce qu'ils sont considérés comme des adultes au regard de la loi ou soit parce que leur placement est terminé, vivent en outre une période critique qui peut nuire à leur développement, soit leur entrée dans l'âge adulte. Selon Arnett et al. (2011), l'entrée dans l'âge adulte requiert un certain nombre d'étapes développementales, comme l'exploration de l'identité et l'expérimentation de rôles, toutes deux étant associées au soutien social de la personne. Plus particulièrement, les jeunes qui sont sur le point de terminer leur placement manquent généralement de relations significatives avec des proches et de réseaux sociaux stables qui contribuent positivement à leur sain développement (Lanctôt, Cernkovich et Girodano, 2007; Lee et Berrick, 2014). 
De plus, ces jeunes n'ont généralement pas toutes les aptitudes nécessaires pour réaliser par eux-mêmes une bonne transition vers l'âge adulte et demeurent ainsi assez dépendants des services de santé mentale offerts aux adultes (Bright et Jonson-Reid, 2010; Corneau et Lanctôt, 2004; Osgood, Foster et Courtney, 2010; McMahon, 2014). Par exemple, des études de suivi effectuées auprès de jeunes femmes ayant été placées à l'adolescence indiquent qu'un tiers d'entre elles ont consulté un psychologue ou un psychiatre au début de l'âge adulte (Corneau et Lanctôt, 2004). Même si de nombreuses études ont analysé les répercussions de l'agression sexuelle, à notre connaissance, aucune ne s'est intéressée à cette étape critique qu'est l'entrée dans l'âge adulte pour des jeunes femmes qui sortent d'un milieu de placement (Zelechoski et al., 2013).

En outre, les recherches ont principalement examiné les effets généraux de l'agression sexuelle sur les symptômes liés au trauma alors que les caractéristiques de l'expérience (par ex. : l'âge de la première survenue de l'agression sexuelle et l'identité de l'agresseur) et leurs impacts relatifs sur les symptômes liés au trauma sont sous-étudiés. Les chercheurs qui ont étudié ces effets spécifiques ont trouvé que les individus ayant rapporté des expériences d'agression sexuelle durant l'adolescence plutôt que durant l'enfance sont dix fois plus à risque de manifester des symptômes sévères de stress post-traumatique (Schoedl et al., 2010). En comparaison, ceux qui ont eu une exposition précoce, durant l'enfance, sont plus à risque de présenter des symptômes de dépression. Ainsi, l'impact de l'agression sexuelle, en termes de symptômes liés au trauma, peut varier selon les stades développementaux (Schoedl et al., 2010). La symptomatologie des traumatismes peut aussi varier selon l'identité de l'agresseur, qu'il soit ou non à l'intérieur du cercle familial. Par exemple, une symptomatologie des traumatismes plus importante a été observée chez des enfants et des adolescents ayant été agressés sexuellement par un membre de la famille nucléaire, comparativement à ceux qui l'ont été par une personne hors du cercle familial (Spaccarrelli et Fuchs, 1997; Thériault, Cyr et Wright, 2003). Plus spécifiquement, l'agression sexuelle de nature incestueuse est généralement liée à des niveaux plus élevés d'anxiété et de dissociation chez les adolescents en contexte de placement (Spaccarelli et Fuchs, 1997; Thériault et al., 2003).

Les répercussions découlant de la fréquence et de la durée de l'agression sexuelle ont été moins étudiées. Monette, Tourigny et Daigneault 
(2008) n'ont pas trouvé de liens significatifs entre la fréquence de l'agression sexuelle et les symptômes liés au trauma. Mais Mclean et al. (2014) ont pour leur part observé plus d'idéations suicidaires chez les adolescents ayant vécu de nombreuses expériences d'agression sexuelle. De plus, la durée de l'agression sexuelle a pu être liée à des niveaux plus élevés de dépression et de préoccupations sexuelles chez les adolescentes en contexte de placement (Thériault et al., 2003).

En résumé, nous en savons peu sur les symptômes liés au trauma se manifestant chez de jeunes femmes ayant été victimes d'agression sexuelle durant l'enfance et ayant par la suite été placées en centre de réadaptation. Une analyse approfondie de la symptomatologie manifestée au début de l'âge adulte pourrait contribuer à une meilleure orientation des programmes et des services. En effet, de récentes études évaluatives indiquent que les programmes sensibles au genre peuvent être plus efficaces pour les femmes que les programmes neutres (Gobeil, Blanchette et Stewart, 2016), en particulier lorsque ces femmes ont rapporté des expériences de négligence et de maltraitance durant l'enfance et manifestent des symptômes liés au trauma (Day, Zahn et Tichavsky, 2015; Saxena, Messina et Grella, 2014). Bien que les recherches reconnaissent clairement le besoin de développer des programmes sensibles au trauma à l'intention des adolescentes ayant des antécédents d'agression sexuelle, il reste toutefois à mieux définir la manière de les mettre en pratique (Kerig et Schindler, 2013). Le premier objectif de cette étude est donc de décrire l'ampleur des symptômes liés au trauma observés au début de l'âge adulte parmi un échantillon de jeunes femmes ayant été placées en centre de réadaptation à l'adolescence, selon que ces jeunes femmes ont rapporté ou non avoir été victimes d'agression sexuelle avant l'âge de 18 ans. Le deuxième objectif consiste à analyser le rôle des caractéristiques de l'agression sexuelle relativement à la symptomatologie présentée par les jeunes femmes ayant été victimes d'agression sexuelle avant l'âge de 18 ans. Ainsi, la sévérité, la variété, la chronicité et l'identité de l'agresseur, la fréquence et l'âge de la première survenue de l'agression sexuelle ont été étudiés en relation avec les symptômes liés au trauma.

\section{Méthodologie}

\section{Participantes}

La présente étude fait partie d'une vaste étude longitudinale auprès d'adolescentes placées en centre de réadaptation dans la grande région 
de Montréal au cours de 2007-2008 (Lanctôt, 2012). Les centres de réadaptation chargés d'offrir les services aux adolescentes sont sous la responsabilité des services de protection de l'enfance du Québec, au Canada. Le placement en centre de réadaptation est considéré comme le dernier recours et est réservé aux jeunes ayant de graves problèmes d'adaptation, ce qui peut affecter leur sécurité et leur développement. Tous les services offerts sont fondés sur les principes de la réadaptation psychosociale et sont assurés par des praticiens qualifiés tels que des psychoéducateurs, des psychologues, des travailleurs sociaux ou des criminologues (Association des centres jeunesse du Québec, 2012). Jusqu'à maintenant, il y a eu six vagues de collectes de données (T1-T6), couvrant la période de la mi-adolescence au début de l'âge adulte. Dans le cadre de la présente étude, seules les données de la sixième vague ont été utilisées. L'échantillon est composé de 133 jeunes femmes adultes (âge moyen $=19,41 ; S D=1,48)$. Elles avaient toutes quitté le centre de réadaptation au moment de l'étude. Le taux d'attrition au temps 6 était de $26 \%$, et ce taux est similaire, voire meilleur, que les taux d'attrition rapportés dans d'autres études conduites avec des individus vulnérables semblables aux jeunes femmes de notre échantillon (Bryan, Schmiege et Magnan, 2012; Moretti, Bartolo, Craig, Slaney et Odgers, 2014).

\section{Procédure de collecte des données}

La procédure de collecte des données a été approuvée par le comité institutionnel d'éthique de la recherche de l'Université de Sherbrooke. Le consentement des participantes a été obtenu lors de chaque vague de collecte de données. Le consentement parental a été obtenu pour toutes les participantes de moins de 14 ans, conformément à l'article $21 \mathrm{du}$ Code civil du Québec. À la suite de l'obtention du consentement, un intervieweur formé en éthique et techniques en recherche a fait passer un questionnaire individuellement à chaque participante. La durée du questionnaire était d'environ 90 minutes. À la fin de l'entretien, on demandait aux participantes de laisser leurs coordonnées afin de pouvoir les contacter pour un suivi.

\section{Mesures}

Agression sexuelle autodéclarée: Ce questionnaire portant sur l'agression sexuelle, qui se fonde sur l'Enquête nationale sur les agressions sexuelles, a été conçu par Finkelhor, Hotaling, Lewis et Smith (1990). Les parti- 
cipantes ont rempli ce questionnaire au cours de la sixième vague de collecte des données. Dans la première partie, les participantes devaient déclarer si elles avaient été victimes ou non d'agression sexuelle avant l'âge de 18 ans, c'est-à-dire si elles avaient été obligées de toucher quelqu'un de manière sexuelle, forcées à être touchées de manière sexuelle ou avoir été violées. La seconde partie portait sur les diverses caractéristiques de l'agression sexuelle, comme la gravité, la diversité des types d'agression, la chronicité, la fréquence, l'âge de la première survenue de l'agression sexuelle et l'identité de l'agresseur. La gravité se rapporte à l'expérience du viol. La diversité comprend les différents types d'agression déclarés et le fait d'avoir été victime de deux types ou plus d'agression (par ex., une combinaison de sévices sexuels et de viol). La chronicité signifie la persistance de l'agression sexuelle au cours des différentes phases de développement de l'enfant, soit 0 à 5 ans, 6 à 12 ans et 13 à 18 ans. En raison du nombre limité de cas, une variable binaire a été créée afin de différencier les occurrences d'agression sexuelle durant une seule phase développementale et les occurrences durant deux phases ou plus. La fréquence se fonde sur le nombre d'expériences d'agression déclarées. Compte tenu de l'écart important observé dans la fréquence, une ligne de démarcation médiane a permis de déterminer deux catégories: de 0 à 4 expériences d'agression sexuelle et 5 et plus. L'âge de survenue réfère à l'âge qu'avait la victime au moment du premier évènement, que ce soit durant l'enfance (0-12 ans) ou l'adolescence (> 12 ans). L'identité de l'agresseur concerne ses caractéristiques. Dans le cadre de l'analyse, les agressions sexuelles ayant eu lieu dans un contexte intrafamilial, où l'agresseur était un membre de la cellule familiale (incluant une combinaison de contextes intra et extrafamiliaux), ont été distinguées des agressions sexuelles ayant eu lieu uniquement dans un contexte extrafamilial.

Symptômes liés au trauma: Les symptômes liés au traumatisme ont été évalués au début de l'âge adulte, lors de la sixième vague de la collecte des données (Trauma Symptom Inventory ${ }^{\mathrm{MC}_{-}} 2-\mathrm{TSI}^{\mathrm{MC}}-2,136$ items, Brière, 2011). Le TSI-2 est un instrument spécifique de mesure du trauma conçu pour évaluer le stress post-traumatique et autres séquelles psychologiques résultant d'évènements traumatiques, y compris les effets de la maltraitance et de la négligence. Quatre grandes échelles de symptômes et leurs indicateurs respectifs ont été évalués: perturbation de soi (symptômes dépressifs, attachement insécure, identité personnelle diffuse), le stress post-traumatique (symptômes anxieux, 
expériences de reviviscence du trauma, évitement défensif, dissociation), l'externalisation (colère, perturbations sexuelles, tendances suicidaires, comportements réducteurs de tension) et la somatisation (problèmes somatiques). Tous les symptômes ont été notés en fonction de la fréquence de leur occurrence dans les six derniers mois, à l'aide d'une échelle de quatre points, allant de «jamais» à «souvent». Les éléments de chaque échelle de symptômes ont été additionnés et les scores les plus élevés représentent les niveaux les plus élevés de symptômes liés au trauma. Le coefficient alpha de Cronbach des quatre facteurs et de leurs indicateurs varie de,84 à,97.

\section{Stratégie d'analyse des données}

Les analyses statistiques ont été réalisées au moyen de la version 20 du logiciel SPSS. Les antécédents d'agression sexuelle et les caractéristiques d'agression sexuelle ont été traités en tant que variables catégorielles alors que les symptômes liés au trauma ont été traités selon une échelle continue. D’abord, une analyse de variance ANOVA a été effectuée pour évaluer les différences dans l'ampleur des symptômes liés au trauma entre les jeunes femmes, selon qu'elles ont rapporté des antécédents d'agression sexuelle ( $n=68 ; 51 \%)$ ou non $(n=65 ; 49 \%)$ ayant survenu avant l'âge de 18 ans. Par la suite, une deuxième série d'analyses a été conduite exclusivement parmi les jeunes femmes ayant rapporté une expérience d'agression sexuelle $(n=68)$ afin d'examiner l'impact de chaque caractéristique relative à l'ampleur des symptômes liés au trauma. Les analyses descriptives ont été effectuées de façon à donner une vue d'ensemble du pourcentage de jeunes femmes ayant rapporté chacune des caractéristiques des expériences d'agression. Ensuite, une analyse de corrélation bisériale de point a été réalisée en vue d'examiner l'interrelation entre les différentes caractéristiques de l'agression sexuelle: la gravité, la diversité du type d'agression, la chronicité, l'identité de l'agresseur, la fréquence et l'âge de survenue de l'agression sexuelle. Les critères de Cohen (1988) ont été utilisés pour interpréter la force des corrélations, selon lesquels $r=0,10-0,29$ a été défini comme faible, $r=0,30-0,49$ modéré et $r>0,50$ fort. Ensuite, une autre série d'ANOVA a été conduite pour examiner les différences dans l'ampleur des symptômes liés au trauma de chaque catégorie: la gravité (viol ou non), la diversité du type d'agression (un type d'agression ou deux et plus), la chronicité (une phase ou deux et plus), l'identité de 
l'agresseur (intrafamilial ou non), la fréquence (moins de 5 ou plus de 5) et l'âge de la première survenue (de 0 à 12 ans ou plus de 12 ans). Les tailles d'effets ( $\mathrm{d}$ de Cohen) ont été calculées pour faciliter l'interprétation de l'ampleur des différences, avec $d=, 20$ considéré comme faible, $d=, 50$, moyen, et $d=, 80$, fort (Cohen, 1988). L'âge au moment de l'évaluation a aussi été inclus dans toutes les analyses en tant que variable contrôle.

\section{Résultats}

Différences des symptômes liés au trauma entre les jeunes femmes ayant été ou non sexuellement agressées avant l'âge de 18 ans

Le Tableau 1 présente une vue d'ensemble des moyennes, des erreurs types et des tailles d'effets de chaque domaine et des symptômes reliés. En somme, des différences significatives ont été observées entre les jeunes femmes avec et sans expérience d'agression sexuelle pour tous les symptômes liés au trauma. La plus grande différence se situe sur le plan des symptômes de stress post-traumatique $(d=1,05)$. Plus précisément, les jeunes femmes ayant des antécédents d'agression sexuelle, comparativement à celles qui n'en ont pas, ont rapporté de plus importants symptômes d'anxiété $(d=, 98)$ et souvenirs sensoriels intrusifs d'un précédent évènement traumatique se manifestant par des cauchemars et des souvenirs (expériences de reviviscence, $d=, 98$ ). De plus, ces jeunes femmes ont rapporté plusieurs tentatives de refoulement ou d'évitement de pensées ou de souvenirs associés à l'agression sexuelle (évitement défensif, $d=1,05)$. Une légère différence a été observée en ce qui concerne la dissociation $(d=, 61)$. Néanmoins, comparativement aux jeunes femmes n'ayant pas rapporté d'expérience d'agression sexuelle, les jeunes femmes avec une telle expérience rapportent encore deux fois plus de symptômes de dissociation, y compris le désengagement, la dépersonnalisation et la déréalisation.

La deuxième différence importante concerne la perturbation de soi $(d=, 71)$, définie comme étant l'incapacité de créer un modèle ou une identité stable de soi. Ce sont les symptômes dépressifs $(d=, 82)$ et l'attachement insécure $(d=, 65)$ qui y contribuent le plus alors que le manque de connaissance de soi et la confusion par rapport à ses pensées et à ses croyances ont une importance moindre (identité diffuse, $d=, 44)$. 
TA B LEA U 1

Différences dans les symptômes liés au trauma (échelles, facteurs) entre les jeunes femmes ayant ou non des antécédents d'agression sexuelle, avec l'âge comme variable contrôle $(n=133)$

\begin{tabular}{|c|c|c|c|c|}
\hline & \multicolumn{2}{|c|}{ Agression sexuelle } & & \\
\hline & Non $(n=65)$ & Oui $(n=68)$ & & \\
\hline & $M(S E)$ & $M(S E)$ & $F$ & $d$ \\
\hline Perturbation de soi & $20,52(17,43)$ & $34,40(19,32)$ & $16,40 * * *$ & ,71 \\
\hline Symptômes dépressifs & $8,51(6,71)$ & $14,57(7,67)$ & $21,61^{* * *}$ & 82 \\
\hline Attachement insécure & $6,05(6,36)$ & $10,75(7,36)$ & $13,69^{* * *}$ &, 65 \\
\hline Identité diffuse & $5,97(6,36)$ & $9,07(6,09)$ & $6,16^{*}$ & 44 \\
\hline Stress post-traumatique & $27,37(22,50)$ & $53,59(25,09)$ & $36,12^{* * *}$ & 1,05 \\
\hline Symptômes anxieux & $7,66(5,42)$ & $13,79(6,60)$ & $31,21^{* * *}$ & 98 \\
\hline Expérience intrusive & $6,88(6,62)$ & $14,46(7,98)$ & $30,92 * * *$ & 98 \\
\hline Évitement défensif & $8,88(8,17)$ & $17,91(8,45)$ & $36,14^{* * *}$ & 1,05 \\
\hline Dissociation & $3,95(4,54)$ & $7,43(5,99)$ & $12,24^{* *}$ & ,61 \\
\hline Externalisation & $18,06(16,26)$ & $31,59(22,77)$ & $12,66^{* *}$ & ,63 \\
\hline Colère & $8,74(6,24)$ & $12,34(6,99)$ & $8,19 * *$ &, 50 \\
\hline Perturbations sexuelles & $2,83(4,57)$ & $6,85(6,70)$ & $13,25^{* * *}$ & ,64 \\
\hline Suicidalité & $2,12(5,15)$ & $4,76(6,92)$ & $4,98^{*}$ & ,39 \\
\hline $\begin{array}{l}\text { Comportement réducteur de } \\
\text { tensions }\end{array}$ & $4,37(4,89)$ & $7,63(6,70)$ & $8,11^{* *}$ &, 50 \\
\hline Somatisation ${ }^{1}$ & $6,18(5,25)$ & $9,60(6,47)$ & $9,86^{* *}$ &, 55 \\
\hline
\end{tabular}

† $\mathrm{p}<, 10,{ }^{*} \mathrm{p}<, 05,{ }^{* *} \mathrm{p}<, 01,{ }^{* * *} \mathrm{p}<, 001$.

1. Le facteur est similaire au score de l'échelle.

L'externalisation $(d=, 65)$ constitue le troisième domaine d'importance associé à l'occurrence d'agression sexuelle. Elle se caractérise par un profil de comportements problématiques, autodestructeurs et agressifs en réponse aux émotions sous-jacentes. Cela se traduit principalement par l'évitement de contact sexuel ou des problèmes de limites au plan sexuel et par un faible contrôle des impulsions sexuelles (perturbations sexuelles, $d=, 64$ ). Les jeunes femmes agressées sexuellement à l'enfance ont aussi rapporté des niveaux plus élevés de cognition, d'humeurs et de comportements colériques (colère, $d=, 50$ ), ainsi que des comportements autodestructeurs et de l'automutilation (comportement de 
réduction des tensions, $d=, 50)$. Les pensées et les comportements suicidaires sont aussi plus fréquents chez les jeunes femmes agressées sexuellement, même si la taille d'effet est moindre $(d=, 39)$. Enfin, les jeunes femmes agressées sexuellement, comparativement à celles n'ayant pas été agressées, étaient plus sujettes à la somatisation $(d=, 55)$.

\section{Interrelations des différentes caractéristiques de l'agression sexuelle envers les mineurs}

Deuxièmement, nous avons examiné les associations entre les caractéristiques des agressions sexuelles (Tableau 2). L'examen des résultats montre des intercorrélations faibles à moyennes pour la majorité des caractéristiques de l'agression sexuelle envers les enfants. Plus d'agressions sexuelles chroniques, ayant eu lieu durant deux phases de développement ou plus, est faiblement associé $(r=, 25 ; p<, 05)$ à une plus large diversité de types d'agression sexuelle (combinaison de sévices sexuels et de viol), et modérément associé $(r=-, 46 ; p<, 01)$ à une survenue précoce de l'agression sexuelle. Ensuite, une plus grande fréquence d'agression sexuelle est modérément associée à une plus large diversité d'expériences d'agression sexuelle $(r=, 39 ; p<, 01)$, à une survenue précoce $(r=-, 36 ; p<, 01)$ et à plus d'agressions sexuelles chroniques $(r=, 43 ; p<, 01)$. L'agression intrafamiliale a été significativement et modérément associée à la survenue de l'agression à un jeune âge (0 à 12) $(r=-, 53 ; p<, 01)$, à plus d'agressions sexuelles chroniques $(r=, 46 ; p<, 01)$ et à un nombre plus élevé d'expériences d'agression sexuelle $(r=, 41 ; p<, 01)$. En dernier, le viol, impliquant la pénétration forcée, a été fortement associé à une plus large variété d'agressions sexuelles $(r=, 81 ; p<, 001)$, et faiblement associé à des agressions sexuelles durant l'enfance plus chroniques $(r=, 32 ; p<, 01)$, et plus fréquentes $(r=, 23 ; p<, 10)$.

\section{Analyses descriptives des types d'agression sexuelle}

Chez les jeunes femmes ayant signalé des expériences d'agression sexuelle $(n=68)$, toutes sauf une ont rapporté avoir été touchées de manière sexuelle $(98,5 \%)$. La seule jeune femme qui n'a pas rapporté avoir été touchée a été forcée de toucher quelqu'un de manière sexuelle. Au total, 51,5\% des jeunes femmes ont rapporté avoir été forcées de toucher quelqu'un de manière sexuelle, et $54,4 \%$ ont rapporté des expériences de viol, impliquant la pénétration forcée. 
TA B LE A U 2

Corrélations entre les caractéristiques de l'agression sexuelle $(n=68)$

\begin{tabular}{|l|c|c|c|c|c|c|}
\hline & 1 & 2 & 3 & 4 & 5 & 6 \\
\hline 1. Diversité & - & & & & & \\
\hline 2. Âge de survenue &,- 04 & - & & & & \\
\hline 3. Chronicité &, $25^{*}$ &,$- 46^{* * *}$ & - & & & \\
\hline 4. Fréquence &, $39^{* *}$ &,$- 36^{* *}$ &, $43^{* *}$ & - & & \\
\hline 5. Intrafamilial &, 16 &,$- 53^{* * *}$ &, $46^{* * *}$ &, $41^{* *}$ & - & - \\
\hline 6. Gravité &, $81^{* * *}$ &, 04 &, $32^{* *}$ &, $23^{\dagger}$ &, 04 & \\
\hline
\end{tabular}

${ }^{\dagger} \mathrm{p}<, 10,{ }^{*} \mathrm{p}<, 05,{ }^{* *} \mathrm{p}<, 01,{ }^{* * *} \mathrm{p}<, 001$.

\section{Le rôle des caractéristiques de l'agression sexuelle}

Un survol des différences dans l'ampleur des symptômes liés au trauma pour chaque caractéristique de l'agression sexuelle est observable dans les Tableaux 3 à 5. La gravité, en termes de viol, contribue marginalement aux symptômes de stress post-traumatique $(d=, 45)$. Les jeunes femmes ayant été violées ont montré plus de symptômes d'anxiété $(d=, 41)$ et de tentatives de répression ou d'évitement de pensées ou de souvenirs associés à l'agression sexuelle (évitement défensif, $d=, 55$ ). Le viol s'est aussi manifesté dans des symptômes plus importants d'attachement insécure $(d=, 59)$.

La diversité des types d'agression sexuelle a également des effets négatifs sur les symptômes post-traumatiques au début de l'âge adulte $(d=, 59)$. Les jeunes femmes qui ont fait l'expérience de deux types d'agression sexuelle ou plus ont montré un plus grand nombre de symptômes d'anxiété $(d=, 59)$, plus d'expériences intrusives $(d=, 59)$ et plus d'évitement défensif $(d=, 67)$. Ces symptômes représentent l'anxiété, le souvenir ou le fait de revivre l'expérience d'agression et l'évitement de stimuli qui déclenchent les émotions. De plus, la diversité des expériences d'agression sexuelle a contribué à l'apparition de symptômes douloureux ou de malaise général plus importants (somatisation, $d=, 54)$. Avoir fait l'expérience de plus d'un type d'agression a un effet marginal sur la perturbation de soi, ce qui se traduit principalement par un attachement insécure $(d=, 59)$. Enfin, les symptômes externalisés ne sont pas significativement associés à la diversité des types d'agression sexuelle. Cependant, un effet marginal a été remarqué pour les comportements réducteurs de tension $(d=, 46)$, montrant ainsi 
TABLEA U 3

Différences de l'ampleur des symptômes liés au trauma (échelles, facteurs) avec l'âge comme variable contrôle: analyse de la gravité et de la diversité des types d'agression sexuelle

\begin{tabular}{|c|c|c|c|c|c|c|c|c|}
\hline & \multicolumn{2}{|r|}{ Gravité } & & & \multicolumn{2}{|c|}{ Diversité des types d'agression sexuelle } & \multirow[b]{3}{*}{ 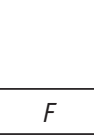 } & \multirow[b]{3}{*}{$d$} \\
\hline & Viol $(n=31)$ & Absence de viol $(n=37)$ & & & 1 type $(n=24)$ & $\geq 2$ types $(n=44)$ & & \\
\hline & $M(S E)$ & $M(S E)$ & $F$ & $d$ & $M(S E)$ & $M(S E)$ & & \\
\hline Perturbation de soi & $31,39(20,17)$ & $36,92(18,47)$ & 1,55 &, 31 & $29,54(19,82)$ & $37,05(18,73)$ & $3,17^{\dagger}$ &, 44 \\
\hline Symptômes dépressifs & $14,10(8,15)$ & $14,97(7,32)$ &, 24 & 12 & $13,25(7,85)$ & $15,30(7,55)$ & 1,34 &, 29 \\
\hline Attachement insécure & $8,58(6,84)$ & $12,57(7,38)$ & $5,57^{*}$ &, 59 & $8,21(6,98)$ & $12,14(7,26)$ & $5,58^{*}$ &, 59 \\
\hline Identité diffuse & $8,71(6,42)$ & $9,38(5,87)$ & 28 & 13 & $8,08(6,33)$ & $9,61(5,95)$ & 1,82 &, 34 \\
\hline Stress post-traumatique & $47,84(25,51)$ & $58,41(24,02)$ & $3,28^{\dagger}$ &, 45 & $44,88(24,30)$ & $58,34(24,49)$ & $5,65^{*}$ &, 59 \\
\hline Symptômes anxieux & $12,39(6,69)$ & $14,97(6,38)$ & $2,78^{\dagger}$ &, 41 & $11,46(6,87)$ & $15,07(6,16)$ & $5,70^{*}$ &, 59 \\
\hline Expérience intrusive & $12,87(7,67)$ & $15,78(8,09)$ & 2,53 &, 40 & $12,25(7,47)$ & $15,66(8,07)$ & $3,84^{*}$ &, 49 \\
\hline Évitement défensif & $15,52(8,82)$ & $19,92(7,67)$ & $4,91^{*}$ &, 55 & $14,46(8,15)$ & $19,80(8,09)$ & $7,23 * *$ & 67 \\
\hline Dissociation & $7,06(6,09)$ & $7,73(5,98)$ & 23 &, 12 & $6,71(5,52)$ & $7,82(6,26)$ & ,74 &, 21 \\
\hline Externalisation & $28,90(23,04)$ & $33,84(12,61)$ & 94 &, 24 & $26,92(23,25)$ & $34,14(22,35)$ & 2,38 &, 38 \\
\hline Colère & $12,55(7,15)$ & $12,16(6,94)$ & 04 &, 05 & $11,96(7,54)$ & $12,55(6,74)$ & 29 &, 14 \\
\hline Perturbations sexuelles & $5,61(6,36)$ & $7,89(6,88)$ & 2,14 &, 36 & $5,67(6,90)$ & $7,50(6,58)$ & 1,59 & ,31 \\
\hline Suicidalité & $4,23(6,78)$ & $5,22(7,10)$ & ,39 &, 16 & $3,33(5,55)$ & $5,55(7,50)$ & 2,11 &, 36 \\
\hline Comportement réducteur de tensions & $6,52(6,66)$ & $8,57(6,69)$ & 1,84 &, 34 & $5,96(6,63)$ & $8,55(6,64)$ & $3,36^{\dagger}$ & 46 \\
\hline Somatisation $^{1}$ & $8,55(5,74)$ & $10,49(6,98)$ & 1,56 & 31 & $7,46(5,18)$ & $10,77(6,85)$ & $4,65^{*}$ &, 54 \\
\hline
\end{tabular}

${ }^{\dagger} p<, 10,{ }^{*} p<, 05,{ }^{* *} p<, 01,{ }^{* * *} p<, 001$.

1. Le facteur est similaire au score de l'échelle. 
T A B LE A U 4

Différences de l'ampleur des symptômes liés au trauma (échelles, facteurs) avec l'âge comme variable contrôle: analyse de la chronicité et de l'identité de l'agresseur

\begin{tabular}{|c|c|c|c|c|c|c|c|c|}
\hline & \multicolumn{2}{|c|}{ Chronicité } & & & \multicolumn{2}{|c|}{ Identité de l'agresseur } & & \\
\hline & $\begin{array}{l}1 \text { phase } \\
(n=46)\end{array}$ & $\begin{array}{c}\geq 2 \text { phases } \\
(n=22)\end{array}$ & & & $\begin{array}{c}\text { Intrafamilial } \\
\quad(n=30)\end{array}$ & $\begin{array}{l}\text { Extrafamilial } \\
\quad(n=38)\end{array}$ & & \\
\hline & $M(S E)$ & $M(S E)$ & $F$ & $d$ & $M(S E)$ & $M(S E)$ & $F$ & $d$ \\
\hline Perturbation de soi & $31,35(18,62)$ & $40,77(19,60)$ & $3,48^{\dagger}$ & 46 & $37,03(18,99)$ & $32,32(19,57)$ & 61 & 19 \\
\hline Symptômes dépressifs & $13,61(7,52)$ & $16,59(7,74)$ & 2,15 &, 36 & $15,47(7,66)$ & $13,87(7,70)$ &, 53 &, 18 \\
\hline Attachement insécure & $9,33(6,89)$ & $13,73(7,58)$ & $5,45^{*}$ &, 58 & $11,47(7,12)$ & $10,18(7,59)$ & 28 &, 13 \\
\hline Identité diffuse & $8,41(5,96)$ & $10,45(6,25)$ & 1,52 &, 31 & $10,10(6,43)$ & $8,26(5,75)$ & 89 & 23 \\
\hline Stress post-traumatique & $48,20(24,07)$ & $64,86(23,88)$ & $6,92^{*}$ &, 65 & $59,63(24,70)$ & $48,82(24,68)$ & 2,63 &, 40 \\
\hline Symptômes anxieux & $13,02(6,56)$ & $15,41(6,55)$ & 1,81 &, 33 & $14,50(6,44)$ & $13,24(6,76)$ &, 38 &, 15 \\
\hline Expérience intrusive & $12,59(7,43)$ & $18,36(7,82)$ & $8,53^{* *}$ & ,72 & $16,73(7,86)$ & $12,66(7,71)$ & $3,79^{\dagger}$ &, 48 \\
\hline Évitement défensif & $15,78(8,46)$ & $22,36(6,59)$ & $9,97 * *$ & ,78 & $20,33(7,48)$ & $16,00(8,77)$ & $4,23^{*}$ &, 51 \\
\hline Dissociation & $6,80(5,43)$ & $8,73(6,98)$ & 1,40 & 29 & $8,07(7,00)$ & $6,92(5,11)$ &, 39 &, 16 \\
\hline Externalisation & $26,70(21,12)$ & $41,82(23,14)$ & $709 *$ &, 66 & $34,37(18,69)$ & $29,39(25,57)$ &, 40 &, 16 \\
\hline Colère & $11,48(6,44)$ & $14,14(7,86)$ & 2,01 & 35 & $13,60(6,61)$ & $11,34(7,20)$ & 1,21 &, 27 \\
\hline Perturbations sexuelles & $5,22(6,01)$ & $10,27(6,90)$ & $9,32^{* *}$ & ,76 & $6,97(5,67)$ & $6,76(7,48)$ & 01 &, 02 \\
\hline Suicidalité & $4,04(6,59)$ & $6,27(7,48)$ & 1,40 & 29 & $4,90(6,45)$ & $4,66(7,35)$ &, 00 &, 02 \\
\hline Comportement réducteur de tensions & $5,96(5,86)$ & $11,14(7,13)$ & $10,10^{* *}$ & ,79 & $8,90(5,85)$ & $6,63(7,23)$ & 1,33 & 29 \\
\hline Somatisation $^{1}$ & $9,52(6,17)$ & $9,77(7,20)$ & 01 & 03 & $10,47(7,05)$ & $8,92(5,98)$ & ,77 & 22 \\
\hline
\end{tabular}

${ }^{\dagger} \mathrm{p}<, 10,{ }^{*}<, 05,{ }^{* *} \mathrm{p}<, 01,{ }^{* * *} \mathrm{p}<, 001$.

1. Le facteur est similaire au score de l'échelle. 
TABLEA U 5

Différences de l'ampleur des symptômes liés au trauma (échelles, facteurs) avec l'âge comme variable contrôle: analyse de la fréquence et de l'âge de survenue des agressions sexuelles

\begin{tabular}{|c|c|c|c|c|c|c|c|c|}
\hline & \multicolumn{2}{|c|}{ Fréquence } & & & \multicolumn{2}{|c|}{ Âge de survenue } & & \\
\hline & $1-4(n=29)$ & $\geq 5(n=34)$ & & & $0-12$ ans $(n=47)$ & $>12$ ans $(n=21)$ & & \\
\hline & $M(S E)$ & $M(S E)$ & $F$ & $d$ & $M(S E)$ & $M(S E)$ & $F$ & $d$ \\
\hline Perturbation de soi & $33,00(20,25)$ & $35,29(18,82)$ &, 29 &, 14 & $36,87(19,14)$ & $28,86(18,99)$ & 1,62 &, 32 \\
\hline Symptômes dépressifs & $14,17(7,39)$ & $14,65(7,72)$ &, 08 &, 08 & $15,28(7,69)$ & $13,00(7,55)$ & ,90 &, 24 \\
\hline Attachement insécure & $9,76(7,94)$ & $11,50(7,05)$ & 97 & 26 & $11,49(7,25)$ & $9,10(7,51)$ & 94 &, 24 \\
\hline Identité diffuse & $9,07(6,65)$ & $9,15(5,93)$ & ,02 &, 03 & $10,11(6,07)$ & $6,76(5,59)$ & $2,92^{\dagger}$ & 42 \\
\hline Stress post-traumatique & $47,41(25,39)$ & $57,91(24,23)$ & $3,22^{\dagger}$ & 46 & $56,09(25,40)$ & $48,00(24,04)$ & 91 & ,24 \\
\hline Symptômes anxieux & $12,90(6,71)$ & $14,38(6,51)$ & 90 & 24 & $14,13(6,43)$ & $13,05(7,09)$ & 14 &, 09 \\
\hline Expérience intrusive & $12,66(7,55)$ & $15,35(8,15)$ & 2,25 &, 39 & $15,49(8,25)$ & $12,14(6,98)$ & 1,65 & 32 \\
\hline Évitement défensif & $14,31(8,26)$ & $20,59(7,44)$ & $10,64^{* *}$ & 84 & $18,53(8,10)$ & $16,52(9,22)$ &, 57 &, 19 \\
\hline Dissociation & $7,55(6,28)$ & $7,59(5,97)$ &, 00 &, 02 & $7,94(6,57)$ & $6,29(4,37)$ & 67 &, 20 \\
\hline Externalisation & $29,17(22,97)$ & $33,71(23,71)$ & ,79 & 23 & $34,38(22,49)$ & $25,33(22,66)$ & 1,29 & 28 \\
\hline Colère & $12,17(6,97)$ & $12,18(7,31)$ &, 00 &, 02 & $12,89(7,10)$ & $11,10(6,72)$ &, 36 &, 15 \\
\hline Perturbations sexuelles & $6,14(6,79)$ & $7,29(6,77)$ &, 53 &, 19 & $7,70(6,51)$ & $4,95(6,88)$ & 1,68 &, 32 \\
\hline Suicidalité & $4,31(6,01)$ & $5,41(7,88)$ &, 48 & 18 & $5,17(7,18)$ & $3,86(6,36)$ & 17 & 10 \\
\hline Comportement réducteur de tensions & $6,55(6,45)$ & $8,82(7,14)$ & 2,16 &, 38 & $8,62(6,85)$ & $5,43(5,94)$ & 2,16 &, 36 \\
\hline Somatisation $^{1}$ & $9,97(6,73)$ & $9,38(6,58)$ & 11 &, 09 & $9,49(6,47)$ & $9,86(6,64)$ & 14 &, 09 \\
\hline
\end{tabular}

${ }^{\dagger} p<, 10,{ }^{*} p<, 05,{ }^{* *} p<, 01,{ }^{* * *} p<, 001$.

1. Le facteur est similaire au score de l'échelle. 
que les jeunes femmes ayant signalé une combinaison de types d'agression sexuelle affichent des niveaux plus élevés de comportements autodestructeurs et d'automutilation au début de l'âge adulte.

La chronicité a visiblement des effets négatifs dans trois domaines sur quatre. Lapport le plus marqué vise le stress post-traumatique $(d=, 65)$. Ces symptômes se traduisent spécifiquement par des niveaux élevés d'expérience intrusive $(d=, 72)$ et d'évitement défensif $(d=, 78)$. Ainsi, les jeunes femmes qui ont eu des occurrences d'agression sexuelle durant deux phases développementales ou plus, comparativement à celles ayant rapporté des agressions sexuelles durant une phase développementale seulement, ont de plus importants symptômes liés aux souvenirs et au fait de revivre les évènements, et une tendance plus marquée à réprimer ou à éviter les pensées ou les souvenirs associés à l'agression sexuelle. La chronicité contribue également aux comportements externalisés $(d=, 58)$, qui se traduisent par des symptômes élevés de perturbation sexuelle $(d=, 76)$ et par des comportements de réduction des tensions $(d=, 79)$. Finalement, la chronicité contribue marginalement à la perturbation de soi $(d=, 46)$, cet effet étant uniquement lié aux symptômes d'attachement insécure élevés $(d=, 58)$. En effet, les jeunes femmes ayant fait l'expérience d'agressions sexuelles durant deux phases de développement ou plus ont rapporté un plus grand nombre d'expériences négatives liées aux figures d'attachement.

L'identité de l'agresseur, en distinguant entre l'agression intrafamiliale et extrafamiliale, a été moins discriminante relativement aux symptômes traumatiques. Il n'y a pas de différences significatives pour les autres domaines. Toutefois, les jeunes femmes qui ont été victimes d'agression intrafamiliale ont montré plus de symptômes liés aux souvenirs ou au fait de revivre l'évènement (expériences intrusives, $d=, 48$ ) et une plus grande tendance à réprimer ou à éviter les pensées ou les souvenirs associés à l'agression (évitement défensif, $d=, 51$ ) que les jeunes femmes ayant été victimes d'agression sexuelle dans un contexte extrafamilial.

La fréquence, en fonction du nombre d'expériences d'agression sexuelle, est marginalement associée aux symptômes de stress posttraumatique $(d=, 46)$. Ces symptômes se traduisent spécifiquement par des symptômes élevés d'évitement défensif $(d=, 84)$. Le nombre d'agressions sexuelles n'est pas indicateur d'autres symptômes élevés observés au début de l'âge adulte. 
L'âge de survenue de l'agression sexuelle, soit durant l'enfance ou l'adolescence, ne s'est pas traduit par des effets différentiels dans les symptômes liés au trauma rapportés au début de l'âge adulte. Nous avons observé un effet marginal exclusivement pour les symptômes qui dénotent une identité diffuse $(d=, 42)$, ce qui montre que les jeunes femmes ayant été agressées sexuellement durant l'enfance ont moins de connaissance de soi et de confiance en soi et manifestent des difficultés à maintenir un soi stable dans leurs relations interpersonnelles.

En somme, la gravité, la diversité des types de maltraitance et la chronicité mènent à des niveaux élevés de symptômes de stress posttraumatiques. Les symptômes se manifestent surtout par des expériences intrusives, de l'attachement insécure et des comportements d'évitement défensif. En comparaison, la fréquence, l'âge de survenue de l'agression sexuelle et l'identité de l'agresseur véhiculent moins d'informations sur les symptômes liés au trauma au début de l'âge adulte chez les jeunes femmes qui ont rapporté avoir été victimes d'agression sexuelle avant l'âge de 18 ans.

\section{Discussion}

L'objectif de cette étude consistait à examiner l'ampleur des symptômes liés au trauma rapportés au début de l'âge adulte parmi un échantillon de jeunes femmes qui ont été placées en centre de réadaptation au cours de l'adolescence et qui ont rapporté avoir été victimes d'agression sexuelle avant l'âge de 18 ans. En vue de mieux comprendre les besoins de ces jeunes femmes et de les transposer dans le cadre de l'intervention, nous avons analysé dans quelle mesure des caractéristiques propres à l'agression sexuelle ont contribué à un certain nombre de symptômes traumatiques. Dans l'ensemble, $51 \%$ des jeunes femmes ayant été placées en centre de réadaptation au cours de leur adolescence ont signalé un historique d'agression sexuelle avant l'âge de 18 ans. Ce résultat correspond à des études antérieures ayant relevé qu'approximativement $60 \%$ des adolescentes en contexte de placement en centre résidentiel avaient eu des expériences d'agression sexuelle (Belknap et Holsinger, 2006; Collin-Vézina et al., 2011; Conner et al., 2004). Plus de 50\% des jeunes femmes ayant signalé une expérience d'agression sexuelle dans le cadre de notre étude ont subi des formes graves d'agression sexuelle, notamment le viol, impliquant la pénétration forcée. 
La recherche montre clairement que les jeunes femmes ayant été placées en centre résidentiel vivent de multiples difficultés au début de l'âge adulte (Corneau et Lanctôt, 2004; Krabbendam et al., 2015; Van der Molen et al., 2013). Ces difficultés se manifestent entre autres par des désavantages socioéconomiques, des relations instables et conflictuelles ainsi que des problèmes de santé mentale (Courtney et al., 2007; Krabbendam et al., 2015; Lanctôt et al., 2007; Lee et Berrick, 2014; McMahon, 2014; Osgood et al., 2010; Van der Molen et al., 2013; Washburn et al., 2007). Nous étude contribue à l'état actuel des connaissances en montrant que parmi un échantillon de jeunes femmes ayant été placées en centre de réadaptation, celles qui ont rapporté des expériences d'agression sexuelle manifestent des niveaux plus élevés de symptômes liés au trauma. En particulier, comparativement aux jeunes femmes de notre échantillon qui n'ont pas rapporté d'agression sexuelle durant l'enfance, celles qui en ont rapporté démontrent une ampleur plus élevée de symptômes en termes de difficultés interpersonnelles et de comportement d'automutilation, ce qui peut indiquer la présence d'un trauma complexe (Brière et Scott, 2015; Cloitre et al., 2009; D’Andrea et al., 2012). De plus, nos résultats montrent qu'une plus grande variété d'expériences d'agression sexuelle ainsi que des formes plus graves et chroniques d'agression sexuelle engendrent une plus importante symptomatologie des traumatismes. Ainsi, une meilleure compréhension en soi de l'agression sexuelle est nécessaire pour mieux comprendre la nature, la gravité et le parcours développemental des symptômes liés au trauma.

Ainsi, les adolescentes placées en centre de réadaptation montrant des caractéristiques relatives à l'expérience d'agression sexuelle doivent recevoir une attention spécifique de la part des intervenants. Bien que nous reconnaissons que les adolescentes ayant été agressées sexuellement sont plus à risque de s'engager dans un cycle de comportements comme la fugue, la prostitution et la consommation de drogue (Kerig et Schindler, 2013; Siegel et Williams, 2003), les interventions doivent être axées sur des cibles d'intervention qui vont au-delà de tels comportements à risque. À cet effet, les interventions fondées sur le trauma sont cruciales. La structure organisationnelle et le cadre de l'intervention doivent favoriser la sensibilité des intervenants à l'égard du trauma, notamment ce qui concerne leurs connaissances des effets de l'expérience traumatique, ainsi que les programmes basés sur des données probantes et adaptés au traitement des expériences traumatiques et des symptômes associés (American Association of Children's Residential Centers, 2014). 
Le modèle d'intervention Attachment, Self-Regulation and Competency (ARC) s'intéresse à trois aspects fondamentaux qui ont des répercussions chez les jeunes ayant un trauma complexe, et qui sont importants pour une résilience future, soit: l'attachement, l'autorégulation et la compétence (Collin-Vézina et Milne, 2014; Hodgdon et al., 2013). Blaustein et Kinniburgh (2015) soulignent la pertinence de cette approche pour soutenir les jeunes en difficulté dans leur transition vers l'âge adulte: «au lieu de considérer la transition qui a cours durant les dernières années de l'adolescence, nous devons nourrir et créer des possibilités de croissance aussitôt que possible» (p. 19).

La première étape de ce modèle préconise le développement de bonnes relations d'attachement avec des intervenants et des proches. Nos résultats soutiennent cette recommandation, les jeunes femmes qui ont été victimes d'agression sexuelle ayant montré de plus grandes difficultés à établir des relations solides, en particulier celles qui ont été exposées à des formes plus graves et chroniques d'agression sexuelle, y compris la pénétration. Dans ce contexte, les programmes d'intervention doivent être sensibles aux besoins des adolescentes et leur fournir un environnement prévisible et sûr. L'importance d'établir un environnement social harmonieux et sécuritaire pour les adolescentes placées en centre de réadaptation est aussi soulevée par Lanctôt, Mathys et Lemieux (2016). Leur étude indique en effet que les adolescentes qui présentent des symptômes liés au trauma plus aigu sont plus enclines à rapporter des perceptions négatives du climat social de leur unité d'hébergement, comme le sentiment d'insécurité ressenti à l'intérieur de leur groupe de pairs. Plus spécifiquement, les interventions fondées sur le traumatisme et axées sur la confiance interpersonnelle et la sécurité semblent cruciales en vue de mieux répondre aux besoins d'un nombre important d'adolescentes placées en centre de réadaptation (Zelechoski et al., 2013).

De plus, les jeunes femmes ayant rapporté des expériences d'agression sexuelle avant 18 ans ont manifesté des symptômes plus importants de stress post-traumatique, parmi lesquels l'anxiété, la reviviscence et l'évitement, mais aussi un plus grand nombre de symptômes externalisés et somatiques. Dans la deuxième phase du modèle ARC, les individus découvrent l'interconnectivité de leurs émotions, de leurs sensations corporelles, de leurs comportements et de leurs cognitions. Ils apprennent également comment l'état d'éveil est connecté à l'évènement traumatique et peut être géré par soi-même ou par le soutien 
sûr des autres. En somme, les interventions doivent être sensibles à la myriade de symptômes que les victimes d'agression sexuelle manifestent, et ne pas seulement répondre aux manifestations de symptômes visibles comme la colère ou l'agressivité, même si ce sont ces symptômes qui sont souvent à l'origine du placement en centre de réadaptation.

La consolidation du concept de soi fait partie des compétences que les jeunes doivent acquérir, notamment lors du passage vers l'âge adulte. Afin d'atteindre cet objectif, l'approche de l'ARC propose de créer des opportunités à l'intention des jeunes et avec elles dans le but d'avoir un pouvoir d'agir sur leur environnement, de découvrir leurs intérêts personnels et leurs forces. Nos résultats vont dans le même sens. En effet, les jeunes femmes ayant rapporté une expérience d'agression sexuelle tendent à avoir une identité personnelle plus diffuse. Ces difficultés se manifestent par une faible connaissance de soi et de la confusion relativement à ses pensées et à ses croyances. À cet égard, Bloom, Owen et Covington (2003) recommandent fortement de renforcer le pouvoir des femmes qui ont été victimes d'agression sexuelle. Le pouvoir d'agir réfère à la capacité de faire des choix déterminés (Sen, 1985) et est une constituante clé de l'autonomisation (Alsop et Heinsohn, 2005; Samman et Santos, 2009). Il ressort de l'étude qualitative réalisée par Hébert, Lanctôt et Turcotte (2016) auprès de jeunes femmes sorties de placement en centre de réadaptation que plusieurs d'entre elles ont ressenti une perte de pouvoir durant leur placement. Les adolescentes placées en centre résidentiel ont souvent l'impression que leur opinion ne compte pas. Selon Munford et Sanders (2015), le pouvoir d'agir des jeunes en difficulté peut être accru par l'établissement d'une relation de confiance avec un adulte au cours de leur placement. Une telle relation peut les aider à faire entendre leur voix au cours des prises de décision les concernant.

Certaines limites doivent être mentionnées. Premièrement, seuls les antécédents d'agression sexuelle ont été examinés dans le cadre de notre étude, même si l'on sait que généralement diverses formes de maltraitance et de négligence peuvent se produire simultanément (Higgins et McCabe, 2000). Par exemple, dans l'étude de CollinVézina et al. (2011) auprès de jeunes placés en centre de réadaptation, plus de la moitié de l'échantillon ont rapporté quatre formes ou plus de mauvais traitement durant l'enfance (y compris l'agression sexuelle). Deuxièmement, même si le questionnaire autorapporté est une méthode adéquate pour étudier les expériences d'agression 
sexuelle et les symptômes liés au trauma, il est possible que les participantes aient eu des difficultés à se remémorer des évènements traumatisants, notamment lorsque ceux-ci se sont déroulés au début de l'enfance (Della Femina, Yager et Lewis, 1990; Hardt et Rutter, 2004). Néanmoins, la recherche a pu montrer que les enfants, dès l'âge de deux ans, peuvent se remémorer des évènements plus tard dans leur vie (Cordon, Pipe, Sayfan, Melinder et Goodman, 2004). En outre, les évènements traumatisants sont généralement plus facilement et plus longuement remémorés que les évènements non traumatisants (Alexander et al., 2005 ; Cordon et al., 2004).

Troisièmement, la présente étude a été conduite parmi un échantillon d'adolescentes ayant été placées en centre de réadaptation, et ainsi, les résultats de cette étude ne peuvent être généralisés à d'autres groupes. De plus, les adolescentes placées en centre de réadaptation sont caractérisées par une myriade de problèmes émotionnels et comportementaux. Ainsi, il est difficile de démêler les effets de l'agression sexuelle des risques que ces adolescentes ont pu cumuler en cours de leur vie.

Quatrièmement, étant donné la petite taille de l'échantillon, nous n'avons pas pu prendre en considération l'interrelation entre les caractéristiques de l'agression sexuelle. Par exemple, les effets de l'agression intrafamiliale peuvent être obscurcis par sa longue durée et sa fréquence, et par conséquent, peuvent être associés à des niveaux de symptômes liés au trauma plus élevés (Tricket et al., 2011). En utilisant une approche multivariée appropriée qui inclut plusieurs caractéristiques des expériences d'agression sexuelle, Mennen et Meadow (1995) ont pu montrer que la gravité, signalée par la pénétration, prédit de façon plus spécifique des niveaux plus élevés de dépression, d'anxiété et d'estime de soi chez un groupe de jeunes filles et d'adolescentes. De futures recherches sur les effets caractéristiques de l'agression sexuelle à l'aide de modèles multivariés sont recommandées. Enfin, étant donné la nature rétrospective des deux méthodes, la causalité n'a pu être établie (Sobel, Buchanan, Butterfield et Jenkins, 2010).

Il est possible que pour certaines jeunes femmes, le questionnaire autorapporté puisse être la première expérience de divulgation de l'agression sexuelle. La divulgation elle-même peut mener à des niveaux plus élevés de symptômes traumatiques, car elle peut susciter des peurs et des incertitudes additionnelles quant à la réponse de l'entourage à cette divulgation (Bonanno, Noll, Putnam, O'Neill et Trickett, 2003). C'est ce que l'on appelle le paradoxe cruel de la divulgation (Harber et 
Pennebaker, 1992). Il est donc recommandé que la recherche regarde au-delà des caractéristiques de l'agression afin d'analyser les répercussions de la divulgation et ses réactions subséquentes.

\section{Références}

Alexander, K. W., Quas, J. A., Goodman, G. S., Ghetti, S., Edelstein, R. S., Redlich, A. D. et Jones, D. P. (2005). Traumatic impact predicts long-term memory for documented child sexual abuse. Psychological science, 16(1), 33-40.

Alsop, R et Heinsohn, N. (2005). Measuring Empowerment in Practice: Structuring Analysis and Framing Indicators. World Bank Policy Working Paper 3510. Washington DC: World Bank.

American Association of Children's Residential Centers. (2014). Trauma-Informed Care in Residential Treatment. Residential Treatment for Cbildren \& Youth, 31(2), 97-104.

Arnett, J. J., Kloep, M., Hendry, L. B. et Tanner, J. L. (2011). Debating emerging adulthood: Stage or process. New York, NY: Oxford University Press.

Association des Centres Jeunesse du Québec (ACJQ). (2012). Rapport d'activité de l'association des centres jeunesse du Québec 2011-2012 [Activity report of the association of Quebec youth centres 2011-2012]. Montréal, Québec: ACJQ.

Belknap, J. et Holsinger, K. (2006). The gendered nature of risk factors for delinquency. Feminist Criminology, 1, 48-71. doi : 10.1177/1557085105282897.

Blaustein, M. E. et Kinniburgh, K. M. (2015). When age doesn't match stage: Challenges and considerations in services for transition-age youth with histories of developmental trauma. Youth, Young Adults, \& Mental Health. Trauma-Informed Care, 29, 17-20.

Bloom, B., Owen, B. et Covington, S. (2003). Gender-responsive strategies. Research, practice and guiding principles for women offenders, 31-48.

Bonanno, G. A., Noll, J. G., Putnam, F. W., O'Neill, M. et Trickett, P. K. (2003). Predicting the willingness to disclose childhood sexual abuse from measures of repressive coping and dissociative tendencies. Child Maltreatment, 8(4), 302-318.

Briere, J. (2011). Trauma Sympton Inventory-2 (TSI-2) manual. Odessa, FL: Psychological Assessment Resources.

Briere, J. (2015). Pain and suffering. A synthesis of buddhist and western approaches to trauma. Dans V. M. Follette, J. Briere, D. Rozelle, J. W. Hopper et D. I. Rome (dir.), Mindfulness-oriented interventions for trauma. Integrating contemplative practices (p. 11-30). New York, NY: The Guilford Press.

Briere, J. et Scott, C. (2015). Complex trauma in adolescents and adults: Effects and treatment. Psychiatric Clinics of North America, 38, 515-27.

Briggs, E. C., Greeson, J. K., Layne, C. M., Fairbank, J. A., Knoverek, A. M. et Pynoos, R. S. (2012). Trauma exposure, psychosocial functioning, and treatment needs of youth in residential care: Preliminary findings from the NCTSN Core Data Set. Journal of Child $\mho$ Adolescent Trauma, 5(1), 1-15. 
Bright, C. L. et Jonson-Reid, M. (2010). Young adult outcomes of juvenile court-involved girls. Journal of Social Service Research, 36, 94-106.

Bryan, A. D., Schmiege, S. J. et Magnan, R. E. (2012). Marijuana use and risky sexual behavior among high-risk adolescents: Trajectories, risk factors, and event-level relationships. Developmental Psychology, 48, 1429-1442. doi: $10.1037 / \mathrm{a} 0027547$.

Cloitre, M., Stolbach, B. C., Herman, J. L., Van der Kolk, B., Pynoos, R., Wang, J. et Petkova, E. (2009). A developmental approach to complex PTSD: Childhood and adult cumulative trauma as predictors of symptom complexity. Journal of Traumatic Stress, 22, 399-408. doi: 10.1002/jts.20444

Cohen, J. (1988). Statistical Power analysis for the behavioral sciences ( $2^{\mathrm{e}}$ éd.). Hillsdale, NJ: Erlbaum.

Collin-Vézina, D., Coleman, K., Milne, L., Sell, J. et Daigneault, I. (2011). Trauma experiences, maltreatment-related impairments, and resilience among child welfare adolescents in residential care. International Journal of Mental Health and Addiction, 9, 577-589.

Collin-Vézina, D., Daigneault, I. et Hébert, M. (2013). Lessons learned from child sexual abuse research: prevalence, outcomes, and preventive strategies. Child and Adolescent Psychiatry and Mental Health, 7(1), 22-30.

Collin-Vézina, D., et Milne, L. (2014). Adolescents en centre de réadaptation: évaluation du trauma. Criminologie, 47(1), 213-245.

Cook, A., Spinazzola, J., Ford, J., Lanktree, C., Blaustein, M., Cloitre, M., ... van der Kolk., B. (2005). Complex Trauma in Children and Adolescents. Psychiatric Annals, 35(5), 390-398.

Connor, D. F., Doerfler, L. A., Toscano, P. F., Volungis, A. M. et Steingard, R. (2004). Characteristics of children and adolescents admitted to a residential treatment center. Journal of Child and Family Studies, 13, 497-510. doi: 0.1023/B : JCFS.0000044730.66750.57.

Cordón, I. M., Pipe, M. E., Sayfan, L., Melinder, A. et Goodman, G. S. (2004). Memory for traumatic experiences in early childhood. Developmental Review, 24(1), 101-132.

Corneau, M. et Lanctôt, N. (2004). Mental health outcomes of adjudicated males and females: the aftermath of juvenile delinquency and problem behavior. Criminal Behaviour and Mental Health, 14, 251-262.

Courtney, M. E., Dworsky, A., Ruth, G., Havlicek, J., Perez, A. et Keller, T. (2007). Midwest evaluation of the adult functioning of former foster youth: outcomes at age 21. Chicago, IL: Chapin Hall Center for Children.

Courtois, C. A. et Ford, J. D. (dir.). (2009). Treating complex traumatic stress disorders: An evidence-based guide. New York, NY: Guilford Press.

Covington, S. et Bloom, B. (2006). Gender responsive treatment and services in correctional settings. Women er Therapy, 29, 9-33. doi: 10.1300/ J015v29n03_02.

D’Andrea, W., Ford, J., Stolbach, B., Spinazzola, J. et van der Kolk, B. A. (2012). Understanding interpersonal trauma in children: why we need a developmentally appropriate trauma diagnosis. American Journal of Orthopsychiatry, 82(2), 187-200. 
Day, J. C., Zahn, M. A. et Tichavsky, L. P. (2014). What works and for whom? The effects of gender responsive programming on girls and boys in secure detention. Journal of Research in Crime Er Delinquency, 52, 93-129.

Della Femina, D., Yeager, C. A. et Lewis, D. O. (1990). Child abuse: Adolescent records vs. adult recall. Child abuse \& neglect, 14(2), 227-231.

Fergusson, D. M., McLeod, G. F. et Horwood, L. J. (2013). Childhood sexual abuse and adult developmental outcomes: Findings from a 30-year longitudinal study in New Zealand. Child abuse er neglect, 37(9), 664-674.

Finkelhor, D., Hotaling, G., Lewis, I. et Smith, C. (1990). Sexual abuse in a national survey of adult men and women: Prevalence, characteristics, and risk factors. Child Abuse or Neglect, 14(1), 19-28.

Finkelhor, D. (2008). Childhood victimization: violence, crime, and abuse in the lives of young people: violence, crime, and abuse in the lives of young people. New York, NY: Oxford University Press.

Finkelhor, D., Ormrod, R.K. et Turner, H.A. (2009). Lifetime assessment of poly-victimization in a national sample of children \& youth. Child Abuse $\mathbb{F}$ Neglect, 33, 403-411.

Gobeil, R., Blanchette, K. et Stewart, L. (2016). A meta-analytic review of correctional interventions for women offenders gender-neutral versus gender-informed approaches. Criminal Justice and Behavior, 43(3), 301-322.

Goodkind, S., Ng, I. et Sarri, R. C. (2006). The impact of sexual abuse in the lives of young women involved or at risk of involvement with the juvenile justice system. Violence agdinst women, 12(5), 456-477.

Hambrick, E. P., Tunno, A. M., Gabrielli, J., Jackson, Y. et Belz, C. (2014). Using multiple informants to assess child maltreatment: concordance between case file and youth self-report. Journal of Aggression, Maltreatment $\mathcal{W}$ Trauma, 23(7), 751-771.

Harber, K. D. et Pennebaker, J. W. (1992). Overcoming traumatic memories. Dans S.A. Christianson (dir.), The Handbook of Emotion and Memory (p. 359388). Hillsdale, NJ : Erlbaum.

Hardt, J. et Rutter, M. (2004). Validity of adult retrospective reports of adverse childhood experiences: review of the evidence. Journal of Child Psychology and Psychiatry, 45(2), 260-273.

Hébert, S. T., Lanctôt, N. et Turcotte, M. (2016). "I didn't want to be moved there": Young women remembering their perceived sense of Agency in the Context of placement instability. Children and Youth Services Review, 70, 229-237.

Herman, J. (1997). Trauma and recovery. The aftermath of violence - from domestic abuse to political terror ( $2^{\mathrm{e}}$ éd.). New York, NY: Basic Books.

Higgins, D. J. et McCabe, M. P. (2000). Multi-type maltreatment and the longterm adjustment of adults. Child Abuse Review, 9, 6-18.

Hillberg, T., Hamilton-Giachritsis, C. et Dixon, L. (2011). Review of metaanalyses on the association between child sexual abuse and adult mental health difficulties: A systematic approach. Trauma, Violence, $\mathfrak{F}$ Abuse, 12(1), 38-49. 
Hodgdon, H. B., Kinniburgh, K., Gabowitz, D., Blaustein, M. E. et Spinazzola, J. (2013). Development and implementation of trauma-informed programming in youth residential treatment centers using the ARC framework. Journal of Family Violence, 28(7), 679-692.

Kerig, P. K. et Schindler, S. R. (2013). Engendering the evidence base: A critical review of the conceptual and empirical foundations of genderresponsive interventions for girls' delinquency. Laws, 2, 244-282.

Kinniburgh, K. M., Blaustein, M. E. et Spinazzola, J. (2005). Attachment, Selfregulation, and competency. A comprehensive intervention framework for children with complex trauma. Psychiatric Annals, 35(5), 424-430.

Krabbendam, A. A., Colins, O. F., Doreleijers, T. A., van der Molen, E., Beekman, A. T. et Vermeiren, R. R. (2015). Personality disorders in previously detained adolescent females: A prospective study. American Journal of Orthopsychiatry, 85(1), 63-71.

Lanctôt, N., Cernkovich, S. A. et Giordano, P. C. (2007). Delinquent behavior, official delinquency and gender: Consequences for adulthood functioning and well-being. Criminology, 45(1), 131-158.

Lanctôt, N. et Lemieux, A. (2012). Expression et régulation de la colère: Les effets d'un programme cognitif-comportemental appliqué à des adolescentes hébergées en centre de réadaptation. Revue de psychoéducation, 41(2), 209-229.

Lanctôt, N., Lemieux, A. et Mathys, C. (2016). The Value of a Safe, Connected Social Climate for Adolescent Girls in Residential Care. Residential Treatment for Children or Youth, 33(3-4), 247-269.

Lathma-Hummer, V. L., Dollard, N., Robst, J. et Armstrong, M. I. (2010). Innovations in implementation of trauma-informed care practices in youth residential treatment: A curriculum for organizational change. Child welfare, 89, 79.

Lee, C. et Berrick, J. D. (2014). Experiences of youth who transition to adulthood out of care: Developing a theoretical framework. Children and Youth Services Review, 46, 78-84.

Maniglio, R. (2009). The impact of child sexual abuse on health: A systematic review of reviews. Clinical Psychology Review, 29, 647-657.

McLean, C. P., Morris, S. H., Conklin, P., Jayawickreme, N. et Foa, E. B. (2014). Trauma characteristics and posttraumatic stress disorder among adolescent survivors of childhood sexual abuse. Journal of Family Violence, 29(5), 559-566.

McMahon, T. J. (2014). Child maltreatment and emerging adulthood past, present, and future. Child maltreatment, 19(3-4) 135-144.

Mennen, F. E. et Meadow, D. (1995). The relationship of abuse characteristics to symptoms in sexually abused girls. Journal of Interpersonal Violence, 10(3), 259-274.

Ministère de la Santé et des Services sociaux. (2007). On a signalé la situation de votre enfant au DJP : Que devez-vous savoir maintenant? Québec: Gouvernement du Québec.

Monette, M. C., Tourigny, M. et Daigneault, I. (2008). Facteurs associés aux problèmes de comportement intériorisés et extériorisés chez des adolescentes agressées sexuellement. Canadian Journal of Behavioural Science/Revue canadienne des sciences du comportement, $40(1), 31$. 
Moretti, M. M., Bartolo, T., Craig, S., Slaney, K. et Odgers, C. (2014). Gender and the transmission of risk: A prospective study of adolescent girls exposed to maternal versus paternal interparental violence. Journal of Research on Adolescence, 24(1), 80-92.

Munford, R. et Sanders, J. (2015). Young people's search for agency: Making sense of their experiences and taking control. Qualitative Social Work, 1-18.

Osgood, D. W., Foster, E. M. et Courtney, M. E. (2010). Vulnerable populations and the transition to adulthood. The Future of Children, 20(1), 209-229.

Pereda, N., Guilera, G., Forns, M. et Gomez-Benito, J. (2009). The prevalence of child sexual abuse in community and student samples: A meta-analysis. Clinical Psychology Review, 29, 328-338. doi : 10.1016/j.cpr.2009.02.007.

Samman, E. et Santos, M. E. (2009). Agency and Empowerment: A review of concepts, indicators and empirical evidence. Oxford, Royaume-Uni: University of Oxford.

Saxena, P., Messina, N. J. et Grella, C. E. (2014). Who benefits from genderresponsive treatment? Accounting for abuse history on longitudinal outcomes for women in prison. Criminal Justice and Behavior, 41, 417-432.

Schoedl, A. F., Costa, M. C. P., Mari, J. J., Mello, M. F., Tyrka, A. R., Carpenter, L. L. et Price, L. H. (2010). The clinical correlates of reported childhood sexual abuse: an association between age at trauma onset and severity of depression and PTSD in adults. Journal of Child Sexual Abuse, 19(2), 156-170.

Sen, A. (1985). Well-being, agency and freedom: the Dewey lectures 1984. The journal of philosopby, 82(4), 169-221.

Siegel, J. A. et Williams, L. M. (2003). The relationship between child sexual abuse and female delinquency and crime: A prospective study. Journal of Research in Crime and Delinquency, 40(1), 71-94.

Sobel, D. M., Buchanan, D. W., Butterfield, J. et Jenkins, O. C. (2010). Interactions between causal models, theories, and social cognitive development. Neural Networks, 23(8), 1060-1071.

Spaccarelli, S. et Fuchs, C. (1997). Variability in symptom expression among sexually abused girls: Developing multivariate models. Journal of Clinical Child Psychology, 26(1), 24-35.

Stoltenborgh, M., van IJzendoorn, M. H., Euser, E. M. et BakermansKranenburg, M. J. (2011). A global perspective on child sexual abuse: meta-analysis of prevalence around the world. Child maltreatment, 16(2), 79-101.

Thériault, C., Cyr, M. et Wright, J. (2003). Facteurs contextuels associés aux symptômes d'adolescentes victimes d'agression sexuelle intrafamiliale. Child Abuse or Neglect, 27(11), 1291-1309.

Trickett, P. K., Noll, J. G. et Putnam, F. W. (2011). The impact of sexual abuse on female development: Lessons from a multigenerational, longitudinal research study. Development and Psychopathology, 23(2), 453-476.

Van der Molen, E., Vermeiren, R. R. J. M., Krabbendam, A. A., Beekman, A. T. F., Doreleijers, T. A. H. et Jansen, L. M. C. (2013). Detained adolescent females' multiple mental health and adjustment problem outcomes in young adulthood. Journal of Child Psychology and Psychiatry, 54(9), 950-957. 
Van Hoof, M. J., van Lang, N. D., Speekenbrink, S., van IJzendoorn, M. H. et Vermeiren, R. R. (2015). Adult Attachment Interview differentiates adolescents with Childhood Sexual Abuse from those with clinical depression and non-clinical controls. Attachment $\mathcal{W}$ Human Development, 17(4), 354-375.

Van Vugt, E., Lanctôt, N., Paquette, G., Collin-Vézina, D. et Lemieux, A. (2014). Girls in residential care: From child maltreatment to trauma-related symptoms in emerging adulthood. Child Abuse $\mathbb{F}$ Neglect, 38(1), 114-122.

Wamser-Nanney, R. et Vandenberg, R. B. (2013). Empirical Support for the Definition of a Complex Trauma Event in Children and Adolescents. Journal of Traumatic Stress, 26, 671-678. doi: 10.1002/jts.21857

Washburn, J. J., Romero, E. G., Welty, L. J., Abram, K. M., Teplin, L. A., McClelland, G. M. et Paskar, L. D. (2007). Development of antisocial personality disorder in detained adolescents: the predictive value of mental disorders. Journal of Consulting and Clinical Psychology, 75, 221-231.

Zelechoski, A. D., Sharma, R., Beserra, K., Miguel, J. L., DeMarco, M. et Spinazzola, J. (2013). Traumatized youth in residential treatment settings: prevalence, clinical presentation, treatment, and policy implications. Journal of Family Violence, 28(7), 639-652.

\title{
How do female adolescents transition out of residential care? Towards a comprehensive understanding of child sexual abuse in relation to trauma-related symptoms
}

\begin{abstract}
Aim: The current study advances our understanding of the level of traumarelated symptoms reported in emerging adulthood among a sample of out-of-care females who experienced child sexual abuse. To obtain a more comprehensive understanding of the experience of sexual abuse, the impact of a number of characteristics was investigated: severity, variety, chronicity, identity of the perpetrator, frequency, and age of onset. Method: The sample was composed of 133 young adult females who had transitioned out of residential care. Sexual abuse was assessed retrospectively using an adjusted version of a sexual abuse survey developed by Finkelhor et al. (1990); trauma-related symptoms were assessed using the Trauma Symptom Inventory-2 (Briere, 2011). Results: Young adult females who had experienced sexual abuse had much higher levels of trauma-related symptoms than those without such a history. Additionally, females who reported more severe forms (rape), various types (variety), and more persistent (chronicity) experiences of sexual abuse showed elevated levels of traumarelated symptoms, such as intrusive thoughts, insecure attachments, and defensive avoidance. Differences in frequency, identity of the perpetrator, and onset were less predictive of the level of trauma-related symptoms. Conclusion: Assessment of the specific characteristics of sexual abuse confirms the need to support females who have experienced persistent and diverse experiences of sexual abuse in developing secure relationships.
\end{abstract}

KEYWORDS - Sexual abuse, trauma-related symptoms, emerging adulthood, females, residential care. 


\section{La transición de las adolescentes afuera de los centros de rehabilitación. Hacia una mayor comprensión del abuso sexual del niño en relación con los sintomas del traumatismo}

RESUMEN - Objetivo: El presente artículo busca enriquecer los conocimientos sobre los sintomas traumáticos observados en la edad adulta en el caso de jóvenes víctimas de violencias sexuales durante la infancia que salen de un centro de rehabilitación. Con el fin de comprender mejor la agresión sexual, estudiamos el impacto de características determinadas como la gravedad, la diversidad de los tipos de abuso, la cronicidad, la identidad del agresor, la frecuencia y la edad en la cual han comenzado los abusos sexuales. Método: la muestra está compuesta por 133 mujeres jóvenes adultas que acaban de regresar de un refugio. El abuso sexual ha sido evaluado retrospectivamente con la ayuda de una versión adaptada del cuestionario sobre el abuso sexual desarrollado por Finkelhor et al. (1990). Los sintomas ligados al traumatismo han sido evaluados con la ayuda del Inventario de los síntomas ligados al traumatismo-2 (Brière, 2011). Resultados: las mujeres jóvenes que vivieron una experiencia de violencia sexual presentan niveles más elevados de síntomas ligados al traumatismo que aquellas que no han sido abusadas sexualmente. Además, las mujeres que señalaron la gravedad de una de las formas de violencia (violación), una diversidad y persistencia (cronicidad) más importante de los abusos sexuales, muestran niveles elevados de sintomas ligados al traumatismo. Dichos síntomas se manifiestan a través de pensamientos intrusivos, problemas del apego, y evitamiento defensivo. Las diferencias observadas en cuanto a la frecuencia, a la identidad del agresor, y a la edad del comienzo de los abusos sexuales son menos discriminativas en lo que atañe a los sintomas ligados al traumatismo. Conclusión: el análisis de las características específicas de los abusos sexuales confirma la necesidad de ayudar a las mujeres que han vivido experiencias persistentes y diversas de abuso sexual con el fin de que puedan desarollar relaciones basadas en el sentimiento de seguridad.

PALABRAS CLAVE - Violencia sexual, síntomas ligados al traumatismo, principio de la edad adulta, mujeres, centro de rehabilitación. 